\title{
Photoproduction of mesons off light nuclei
}

\author{
B. Krusche ${ }^{* \dagger}$ \\ Department of Physics, University of Basel, Switzerland \\ E-mail: Bernd.Krusche@unibas.ch
}

\begin{abstract}
Recent results for the photoprodcution of mesons of light nuclei $\left({ }^{2} \mathrm{H},{ }^{3} \mathrm{He}\right.$, and $\left.{ }^{7} \mathrm{Li}\right)$ are summarized. This series of experiments was run at the electron accelerators ELSA in Bonn and MAMI in Mainz, using tagged photon beams and almost $4 \pi$ covering electromagnetic calorimeters as detectors. It covers two main topics. The investigation of meson production reactions off quasi-free neutrons is used as a tool for the study of the electromagnetic excitation spectrum of the neutron. Total cross sections, angular distributions, and polarization observables have been studied for different reactions and systematic effects arising from the quasi-free nature of the reactions have been carefully investigated. These experiments used mainly deuterium targets, but also ${ }^{3} \mathrm{He}$ for systematic cross-checks of nuclear effects. Some examples for $\eta$ and double-pion production will be discussed. Photoproduction off nuclei was furthermore explored for the study of hadron in-medium properties and the interaction of mesons with nuclei. This program extends to heavier nuclei, but here we limit the discussion to the search for $\eta$-mesic states in helium and lithium nuclei.
\end{abstract}

Sixth International Conference on Quarks and Nuclear Physics,

April 16-20, 2012

Ecole Polytechnique, Palaiseau, Paris

*Speaker.

${ }^{\dagger}$ for the CBELSA/TAPS, Crystal Ball, and TAPS collaborations 


\section{Introduction}

Photoproduction of mesons off nuclei is related to many different topics. As sketched in Fig. 1 it can be classified into three different main processes. In breakup reactions at least one nucleon is removed from the nucleus. A limiting case are quasi-free reactions, where the meson is produced on a participant nucleon in quasi-free kinematics (i.e. like on a free nucleon apart from the offshell momentum of the bound nucleon), the nucleon is kicked out from the nucleus and the rest nucleus may be regarded as a spectator. This reaction type is explored for measurements of meson production reactions from quasi-free neutrons, in particular with deuteron targets. For heavier target nuclei it is also used to study hadron in-medium properties and meson - nucleon interactions (see e.g. $[1,2,3,4])$. The other extreme are coherent reactions, where the meson is produced in a
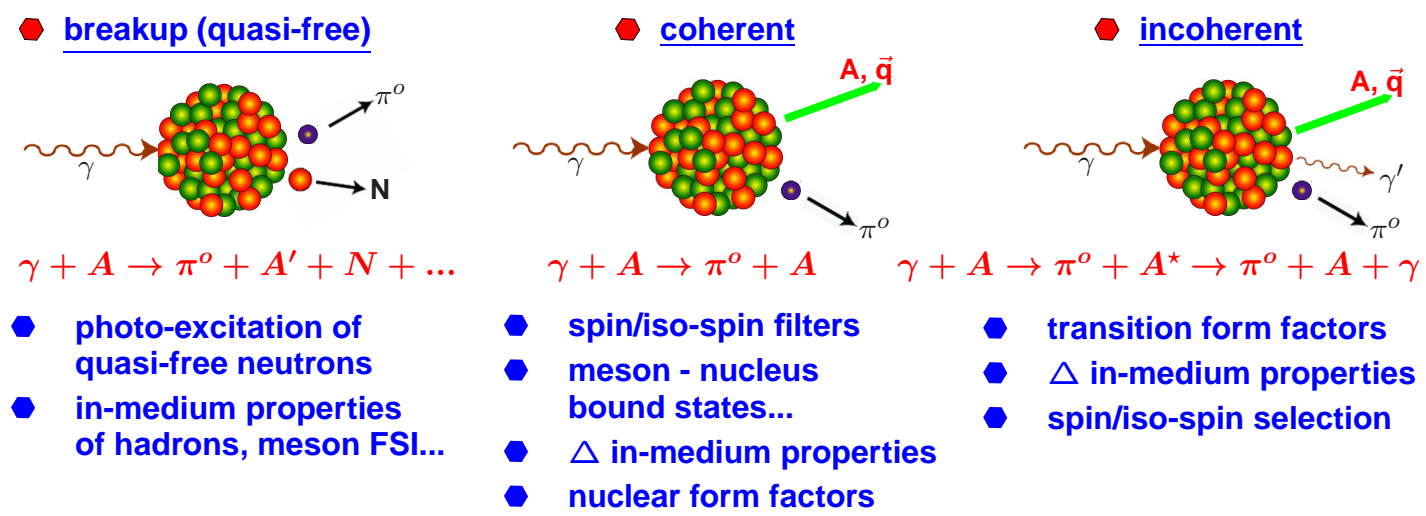

Figure 1: Different nuclear meson production processes for the example of single $\pi^{0}$-photoproduction.

coherent superposition of the amplitudes from all nucleons and the final state nucleus remains in its ground state. This reaction type may be used to filter specific spin/isospin components of the elementary reaction amplitudes. It is also used for the study of hadron-in-medium properties like the in-medium width and mass of the $\Delta$-resonance [5]. Even applications for nuclear properties like nuclear mass-form-factors [6] have been discussed. Incoherent production to excited nuclear states adds further degrees of freedom by the selection of the quantum numbers of the final state (allowing for different filters for the elementary amplitudes or the measurement of transition form factors). However, the cross sections are quite small so that so far almost only $\pi^{0}$-production in the $\Delta$-resonance region has been considered [7]. A recent overview over the experimental photonuclear programs at the involved accelerator laboratories is given in [8]. Here we concentrate on two topics of photoproduction from light nuclei.

Photoproduction of mesons off the free proton has been studied during the last few years with large experimental programs measuring angular distributions, single, and double polarization observables in particular at the tagged photon beams of ELSA (Bonn), Jlab (Newport News), MAMI (Mainz), and until recently also at ESRF (Grenoble) in an attempt to clarify the unsolved issues of the nucleon excitation spectrum like the 'missing resonance' problem. However, a full classification of this spectrum is impossible without the isospin dependence of the electromagnetic nucleon excitations, which require the measurement of photoproduction reactions off neutrons. This program is still much less developed than the $\gamma p$ measurements. Reactions off neutrons can be only 
measured in quasi-free mode from neutrons bound in light nuclei, in particular the deuteron. This introduces additional problems into the measurements (coincident detection of recoil neutrons), as well as into the interpretation of the data (Fermi motion, nuclear Final State Interaction (FSI) effects). During the last few years our group has started an intensive program to measure such reactions at the ELSA and MAMI facilities. The problems involved in the measurement and interpretation of of quasi-free reactions have been systematically studied. First results have been published for $\eta$ and $\eta^{\prime}$ production $[9,10,11]$ and many more results are in preparation.

The other topic is meson-nucleus interaction. A long standing question, that has been again controversially discussed recently, is whether the strong interaction can produce (quasi)-bound meson-nucleus states. Most promising candidates are $\eta$-mesic states due to the strong s-wave $\eta$ nucleon interaction at small $\eta$ momenta. This interaction arises from the very large coupling of the $\eta N$ system to the $\mathrm{S}_{11}(1535)$ nucleon resonance $[12,13]$. One experimental approach to this problem is the study of coherent photoproduction of $\eta$-mesons from light nuclei. The existence of quasi-bound states should give rise to a strong enhancement of the threshold production cross section over phase-space expectation. Results for ${ }^{3} \mathrm{He}[14]$ have just been published and results for ${ }^{7} \mathrm{Li}$ are in preparation for publication [15].

\section{Experiments}

The experiments have been done and are still running at the tagged photon facilities of the Bonn ELSA [16] and Mainz MAMI [17, 19] accelerators. Both experiments use electromagnetic calorimeters covering solid angles of almost $4 \pi$ which are shown in Fig. 2. In the setup in Mainz the targets are surrounded by the Crystal Ball detector [21], which is made of 672 $\mathrm{NaI}$ modules. It is combined with a forward wall made of $\mathrm{BaF}_{2}$ crystals from the TAPS detector [22] (different versions were in use, one with 510 modules, on with 384 modules). Details are given in [18]. The setup used at ELSA combines the Crystal Barrel detector (1290 CsI(Tl) crystals) [20] also with a forward wall of TAPS crystals. The version

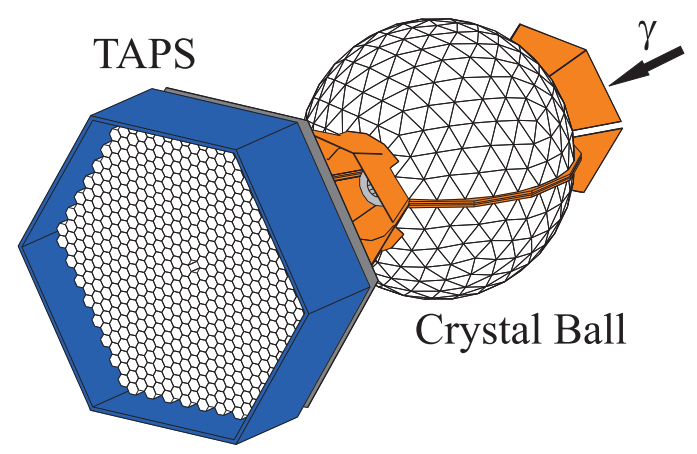
used for the already published results is discussed in [11], the most recent version is shown in Fig. 2. Mini-TAPS

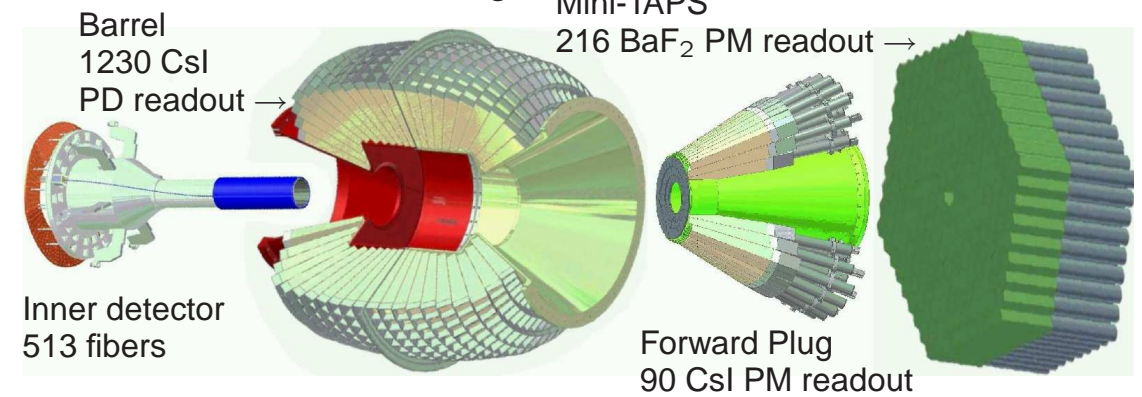

Figure 2: Upper part: Schematic drawing of the Crystal Ball/TAPS calorimeter at MAMI. Bottom part: Schematic setup of Crystal Barrel/TAPS experiment at ELSA 


\section{Results}

\subsection{Photoproduction of mesons off the neutron}

This program requires a reliable detection of recoil neutrons and a precise control of their detection efficiency. Both detector systems are capable to detect neutrons with typical detection efficiencies between 5\% - 30\%, depending on their kinetic energies. The efficiencies can be simulated rather reliably with program packages like GEANT-CALOR [23] and the simulations can be cross-checked with data for example from the $\gamma p \rightarrow n \pi^{0} \pi^{+}$reaction. Furthermore, for most reactions of interest, the cross section for coherent production off the deuteron is very small (or in the few other cases like single $\pi^{0}$ production at low energies also measurable) so that the condition:

$$
\sigma_{\text {incl }}=\sigma_{p}+\sigma_{n}\left(+\sigma_{d}\right)
$$

allows for a consistency check. Here, $\sigma_{p}, \sigma_{n}$ are the quasi-free cross sections measured via detection of coincident recoil nucleons, $\sigma_{\text {incl }}$ is the inclusive cross section measured without any condition for recoil nucleons (may be detected or not), and $\sigma_{d}$ is coherent production off the deuteron. In principle, Eq. 3.1 gives even the possibility to measure neutron cross sections without detection of recoil neutrons via $\sigma_{n} \approx \sigma_{i n c l}-\sigma_{p}$. Details of such analyses are given in $[9,10,11]$.
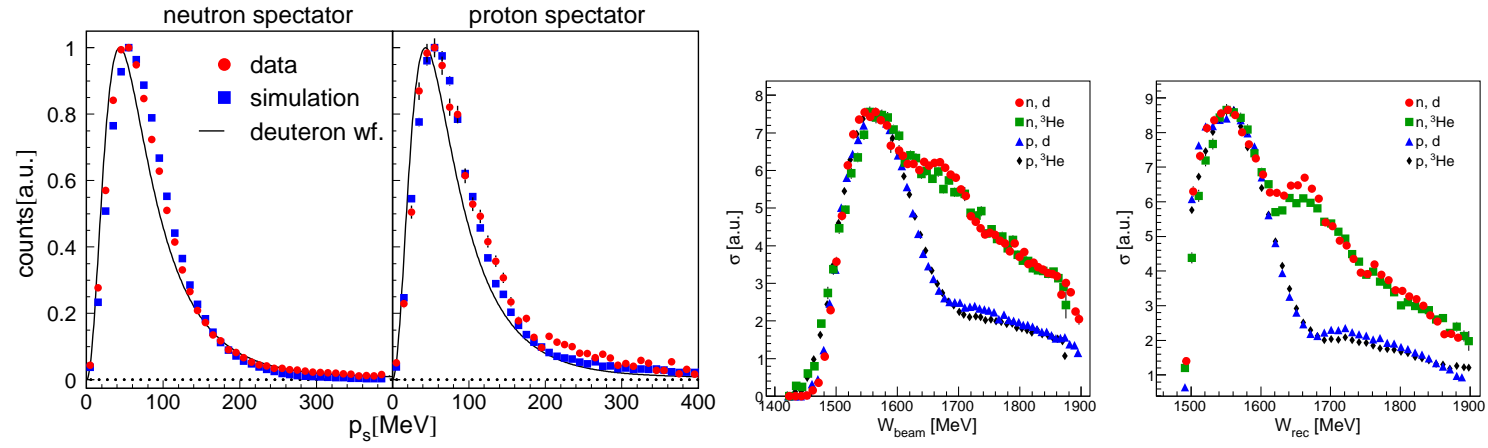

Figure 3: Left hand side: Momentum distributions of spectator nucleons from $\gamma d \rightarrow n p \eta$. (Red) dots: reconstructed from data, (black) lines: expected from deuteron wave function [24], (blue) squares: Monte Carlo simulation including detector response. Neutron (proton) spectator means proton (neutron) detected. Right hand side: preliminary excitation functions for $\gamma d \rightarrow(n)(p) \eta$ and $\gamma^{3} \mathrm{He} \rightarrow(n)(p) \eta X$ measured at MAMI. $W_{\text {beam }}$ from incident photon energy, $W_{\text {rec }}$ from kinematic reconstruction.

Data measured from nucleons bound in light nuclei is at least smeared out by the momentum distribution of the off-shell nucleons and may also be influenced by Final State Interaction (FSI) effects. However, when the recoil nucleons are detected in coincidence, at least for the deuteron, the final state is kinematical completely determined and the 'true' $W$ of the photon - nucleon (respectively nucleon - meson) pair can be extracted. In both detector systems, kinetic energies of the recoil neutrons can only be measured with time-of-flight methods and are therefore limited to neutrons detected in the TAPS forward walls (flight distance is too short for the Crystal Ball or Barrel). However, for the deuteron target, the final state is already completely determined when only the direction of the neutron is measured [11, 8]. This is demonstrated in Fig. 3. The momentum distributions of the spectator nucleons agree very well with a Monte Carlo simulation using the 
deuteron wave-function [24] as input. This shows also, that nuclear effects beyond Fermi motion are not important. As an additional check one can then compare the reconstructed quasi-free proton excitation function to free proton data. Excellent agreement was found for $\eta$ production [11]. These analyses are the basis for the results that show a pronounced structure around $W=1.67 \mathrm{GeV}$ in the $\eta$-neutron excitation function $[9,11]$, the nature of which is still under discussion. The right hand side of Fig. 3 shows preliminary results for quasi-free $\eta$ excitation functions off quasi-free protons and neutrons from the deuteron and from ${ }^{3} \mathrm{He}$ measured at MAMI [25]. From both measurements the structure is clearly visible. The resolution of the reconstructed $W$ is better for the deuteron, since it involves an approximation (negligible relative momentum of spectator nucleons) for the four-body final state in the helium case.

In the next generation of experiments (which was already started at both facilities) single and double polarization observables are being measured, the latter using a deuterated frozen-spin buthanol target. As one example we show preliminary results for a single polarization observable, the beam-helicity asymmetry $I^{\odot}(\Phi)$ in double $\pi^{0}$ production. It is defined for three-body final states as the count-rate difference measured between the two circular polarization states of the photon beam as function of the angle $\Phi$ defined in Fig. 4 between reaction and production plane. This observable is particularly sensitive to small interference terms between different photoproduction multipoles. Previous results for the three isospin final states $p \pi^{0} \pi^{0}, n \pi^{0} \pi^{+}$, and $p \pi^{+} \pi^{-}$, measured off free protons, revealed substantial problems for models of these reactions $[26,27,18]$. Fig. 5 shows preliminary results for

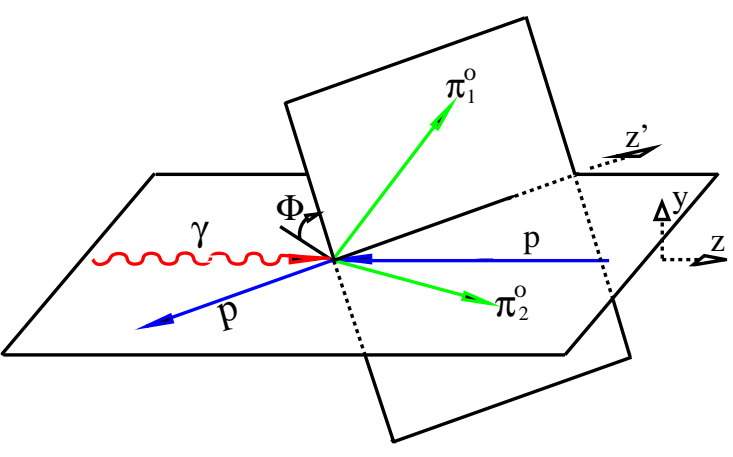

Figure 4: Definition of the angle $\Phi$ $2 \pi^{0}$ production off quasi-free nucleons.
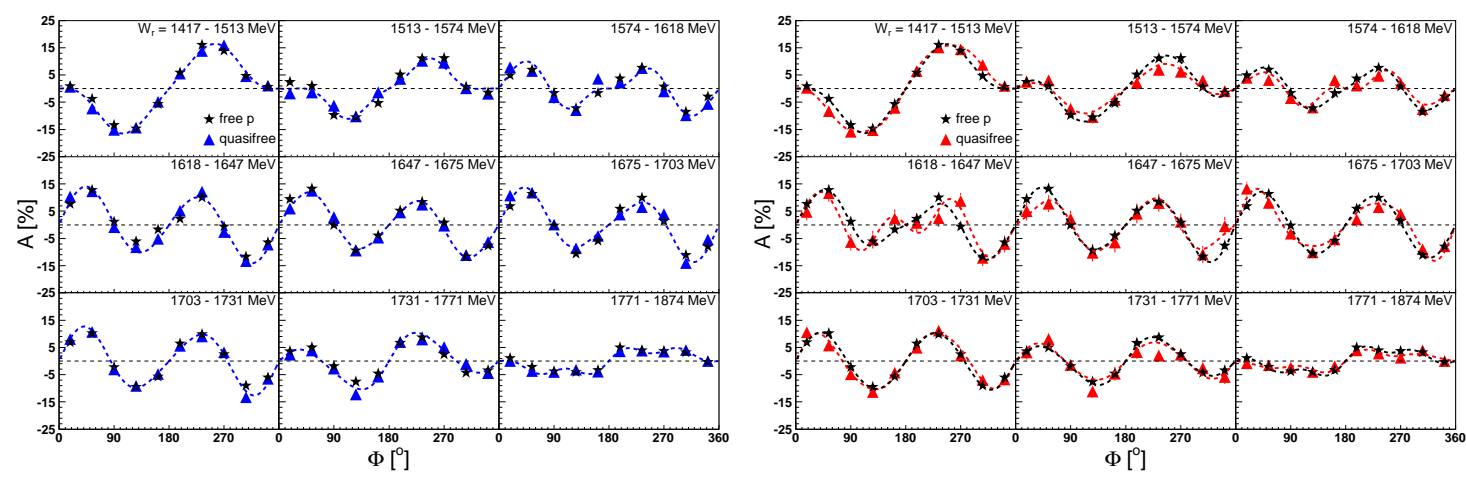

Figure 5: Left hand side: beam helicity asymmetry for free (black stars) and quasi-free (blue triangles) protons. Right hand side: Same for quasi-free neutrons (red triangles) compared to free protons (preliminary).

The data have been measured with a liquid deuterium target at MAMI. Different from the results in $[27,18]$ the two neutral pions have been ordered by the pion-proton invariant masses such that $m\left(\pi_{1}^{0}, p\right) \geq m\left(\pi_{2}^{0}, p\right)$, so that also odd components contribute to the asymmetries. Also for this observable, excellent agreement is obtained between the results for free and quasi-free protons 
(left hand side of Fig. 5) after kinematic reconstruction of the final state. This is far from trivial since the Fermi motion also tends to smear-out the involved angles. The surprising result of this measurement is that the observed asymmetries are almost identical for protons and neutrons. Since at least for the higher incident photon energies models indicate different resonance contributions this was not expected.

\subsection{Search for $\eta$-mesic states}

The threshold behavior of different $\eta$-production reactions has been intensively studied during the last few years in the search for $\eta$-mesic states. Apart from hadron induced reactions, photon induced coherent production was investigated. Unfortunately, this process has very small cross sections for most nuclei. Since it is dominated by the isovector, spin-flip excitation of the $S_{11}(1535)$ resonance [28], only nuclei with ground state spin and isospin different from zero are promising targets. Among the lightest stable nuclei, this condition is fulfilled for ${ }^{3} \mathrm{H},{ }^{3} \mathrm{He},{ }^{7} \mathrm{Li},{ }^{9} \mathrm{Be}$. The $\gamma^{3} \mathrm{He} \rightarrow \eta^{3} \mathrm{He}$ reaction had been previously studied by Pfeiffer et al. [29] with the TAPS detector at MAMI. A strong enhancement of the coherent reaction close to threshold was reported, but the statistical quality of the data was limited. A new measurement with the combined Crystal Ball/TAPS setup has improved the results considerably [14]. As shown at the left hand side of Fig. 6 the total cross section rises abruptly at threshold, far steeper than expected from reaction phase-space obtained with a PWIA modelling. At the same time, angular distributions close to threshold are much flatter [14] than expected from the behavior of the nuclear form factor. Both
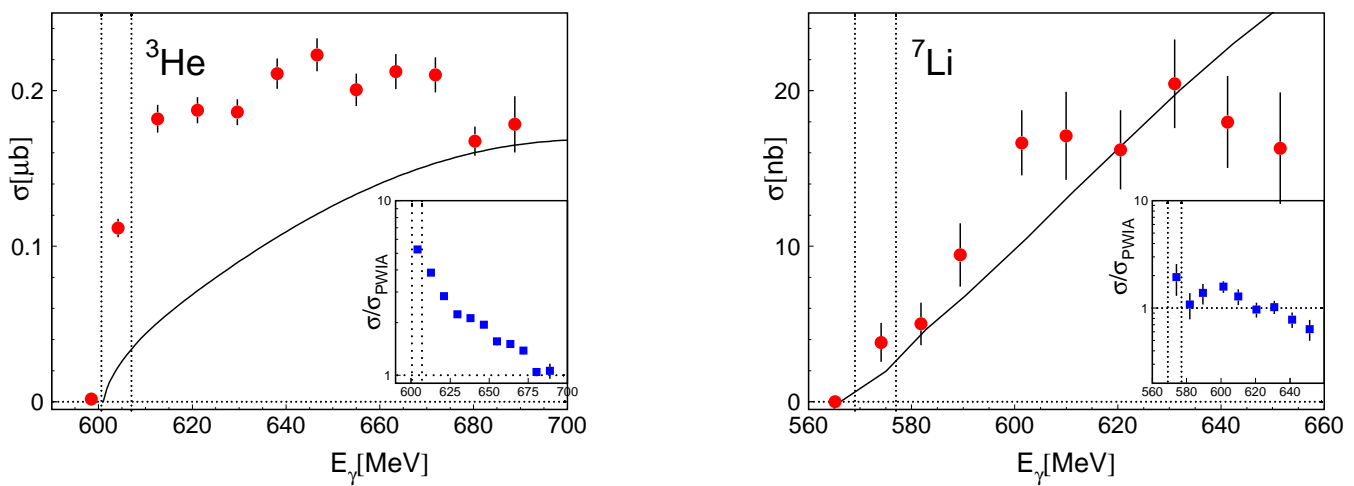

Figure 6: Left hand side: Total cross section for the $\gamma^{3} \mathrm{He} \rightarrow \eta^{3} \mathrm{He}$ reaction compared to plane-wave impulse approximation [14]. Vertical dashed lines indicate coherent and breakup threshold for $\eta$-production. Insert: ratio of data and impulse approximation. Right hand side: same for ${ }^{7} \mathrm{Li}$ [15].

results are in line with a resonant structure close to threshold. However, so far almost nothing is known experimentally for the systematics of coherent $\eta$-production. A measurement with a tritium target would be desirable, but cannot be easily done due to the specific target problems. Very recently, the first attempt was made to measure this reaction with a ${ }^{7} \mathrm{Li}$ target at the MAMI setup. The observed total cross section is roughly one order of magnitude smaller than for ${ }^{3} \mathrm{He}$. This is simply due to the nuclear form factor, which is much steeper. Preliminary results are shown at the right hand side of Fig. 6. The threshold behavior of the cross section is in reasonable agreement with a PWIA approximation, indicating no need for resonant processes. This result further highlights the special role of the ${ }^{3} \mathrm{He}-\eta$ system. 


\section{Acknowledgments}

The discussed experiments are part of the experimental program of the Crystal Ball/TAPS (Mainz) and Crystal Barrel/TAPS (Bonn) collaborations. The data have been obtained in the context of $\mathrm{PhD}$ theses works of I. Jaegle, Y. Magrbhi, M. Oberle, F. Pheron, D. Werthmüller, and L. Witthauer. This work was supported by Schweizerischer Nationalfonds and Deutsche Forschungsgemeinschaft (SFB/TR-16.)

\section{References}

[1] J.G. Messchendorp et al., Phys. Rev. Lett. 89222302 (2002).

[2] F. Bloch et al., Eur. Phys. J. A 32219 (2007).

[3] T. Mertens et al., Eur. Phys. J. A 38195 (2008).

[4] M. Nanova et al., Phys. Lett. B 710600 (2012)

[5] B. Krusche et al., Phys. Lett. B 526287 (2002).

[6] B. Krusche, Eur. Phys. J A 267 (2005).

[7] C.M. Tarbert et al., Phys. Rev. Lett. 100132301 (2008).

[8] B. Krusche, Eur. Phys. J. Special Topics 198, 199 (2011).

[9] I. Jaegle et al., Phys. Rev. Lett. 100, 252002 (2008).

[10] I. Jaegle et al., Eur. Phys. J. A 47, 11 (2011).

[11] I. Jaegle et al., Eur. Phys. J. A 47, 89 (2011).

[12] B. Krusche et al., Phys. Rev. Lett. 74, 3736 (1995).

[13] B. Krusche et al., Phys. Lett. B 397, 171 (1997).

[14] F. Pheron et al., Phys. Lett. B 709, 21 (2012).

[15] Y. Maghrbi et al., in preparation (2012).

[16] W. Hillert, Eur. Phys. J. A 28, 139 (2006).

[17] Th. Walcher, Prog. Part. Nucl. Phys. 24, 189 (1990).

[18] F. Zehr et al., Eur. Phys. J. A 48, 98 (2012)

[19] S.J. Hall, G.J. Miller, R. Beck, P.Jennewein, Nucl. Inst. and Meth. A 368, 698 (1996).

[20] E. Aker et al., Nucl. Instr. and Meth. A 321, 69 (1992).

[21] A. Starostin et al., Phys. Rev. C 64, 055205 (2001).

[22] A.R. Gabler et al., Nucl. Inst. and Meth. A 346, 168 (1994).

[23] C. Zeitnitz et al., The GEANT-CALOR interface user's guide (2001). (http://www.staff.uni-mainz.de/zeitnitz/Gcalor/gcalor.html)

[24] M. Lacombe et al., Phys. Lett. B 101, 139 (1981).

[25] D. Werthmüller, L. Witthauer et al., in preparation (2012).

[26] S. Strauch et al., Phys. Rev. Lett. 95, 162003 (2005).

[27] D. Krambrich et al., Phys. Rev. Lett. 103, 052002 (2009).

[28] B. Krusche and S. Schadmand, Prog. Part. Nucl. Phys. 51, 399 (2003).

[29] M. Pfeiffer et al., Phys. Rev. Lett. 92, 252001 (2004). 